\title{
LA INFORMACIÓN SOBRE RESPONSABILIDAD SOCIAL CORPORATIVA EN LOS HOTELES DE LAS ISLAS CANARIAS
}

\author{
José Juan Déniz Mayor \\ María Concepción Verona Martel \\ José Juan Barrera Rodríguez. \\ Universidad de Las Palmas de Gran Canaria
}

\section{RESUMEN}

En el presente trabajo se ofrece un diagnóstico preliminar acerca de la comunicación sobre responsabilidad social corporativa (RSC) a través de internet por parte de empresas de alojamiento turístico domiciliadas en las Islas Canarias (España) y su relación con determinadas variables societarias y contables. Destaca que las empresas de mayor tamaño, que operan como sociedades anónimas o forman parte de un grupo empresarial son más propensas a difundir datos sobre RSC. Unas cifras superiores en indicadores de rendimiento como el margen neto de explotación, el ROA o el ROE también arrojan resultados significativos. La categoría de los establecimientos propiedad de la sociedad, su longevidad o el endeudamiento no parecen influir en la decisión de publicar.

Palabras clave: Responsabilidad Social Corporativa, Hoteles, Internet.

\section{Disclosing information on corporate social responsibility in Canary Islands hotels}

\section{ABSTRACT}

A preliminary diagnosis about communication on corporate social responsibility (CSR) on the internet by hospitality companies domiciled in the Canary Islands (Spain) and their relationship with certain corporate and accounting variables is provided in this paper. It

Recibido: 4 de abril de 2016

Devuelto para su revisión: 7 de febrero de 2017

Aceptado: 18 de abril de 2017

Grupo de Investigación de Finanzas Cuantitativas y Computacionales. Facultad de Economía, Empresa y Turismo. Universidad de Las Palmas de Gran Canaria. Campus de Tafira. 35017 LAS PALMAS DE GRAN CANARIA (España).E-mail: josejuan.deniz@ulpgc.es, mariaconcepcion.verona@ulpgc.es 
highlights that biggest companies, operating as public limited companies or as a part of a business group are more likely to disseminate data on CSR. Higher values on performance indicators such as net operating margin, ROA or ROE also result in significant results. The category of establishments owned by the company, its age or debt ratio do not appear to influence the decision to publish information on CSR.

Keywords: Corporate social responsibilty, Hotels, Internet.

\section{INTRODUCCIÓN}

De acuerdo con los datos de la Organización Mundial del Turismo (World Tourism Organization (2016), España fue el tercer destino turístico mundial en 2013 con un total de 60,7 millones de visitantes y el segundo por volumen de ingresos turísticos por valor de 62,56 millones de dólares USA. Dentro del territorio nacional, las Islas Canarias recibieron 10,6 millones de visitantes. Esto quiere decir que, si esta región fuese analizada como un país independiente, ocuparía la posición 24 en el ranking mundial de un total de 141 naciones. Ahora bien, aunque el mercado turístico español ha experimentado un crecimiento espectacular, éste se habría debido principalmente a la inestabilidad política y social en muchos países árabes de la cuenca mediterránea que desvía a los viajeros especialmente a las Islas Canarias, haciendo del archipiélago una especie de "valor refugio" para el turismo (Consejo Económico y Social de Canarias, 2013). Por tal motivo, y dado que el sector turístico es clave tanto en la economía estatal como para la canaria, en diferentes ámbitos se considera esencial la implantación de políticas de RSC como elemento diferenciador frente a alternativas competidoras (Guerra y Fernández, 2011). Así, desde el Gobierno de España (Ministerio de Industria, Turismo y Comercio, 2007) se ha planteado como objetivo que el sector turístico nacional sea líder en competitividad y sostenibilidad, aportando a la vez el mayor bienestar social a la población.

Aunque se ha constatado un creciente grado de desarrollo de las políticas de responsabilidad social corporativa (RSC) en el mundo (Albareda y Balaguer, 2008), esta expansión se ha visto seriamente frenada por su insuficiente y deficitaria implementación en las pequeñas y medianas empresas (PYMES) (Forética, 2011). En este sentido y teniendo presente que la implantación de políticas de RSC depende del sector (Brammer y Millington, 2005; Lee y Park, 2009), el objetivo del presente trabajo es identificar las variables que influyen en la comunicación del desempeño socialmente responsable en el sector turístico. Para ello se tendrá presente la información difundida a terceros a través de las páginas de internet corporativas, buscando patrones que permitan establecer relaciones entre dicha información y determinadas características económico-financieras de las empresas consideradas. De este modo, se ha podido comprobar el flujo de comunicación de la empresa sobre temas de RSC.

Ahora bien, centrar el análisis en un canal de comunicación específico, como es internet, conlleva la exclusión de empresas que, realizando acciones en materia de RSC, no utilicen dicho medio y hayan optado por divulgarlas por otras vías, como memorias y folletos impresos o comunicados de prensa o, simplemente, por no informar al respecto. 
No obstante, la razón de utilizar las páginas de internet radica en que son medios de comunicación mucho más accesibles y cercanos a la mayoría del público, en especial los potenciales clientes, que otras posibles vías alternativas, cuya extensión pudiera ser más restringida.

Dentro del sector turístico, la investigación se ha centrado en las empresas de alojamiento. La importancia de este sector en las Islas Canarias queda puesta de manifiesto en que, según Exceltur (Alianza para la Excelencia Turística, 2014), ha llegado a generar en 2013 el 31,2\% del PIB total de Canarias y el 35,2\% del empleo regional. A juicio de esta entidad, estos datos demostrarían la condición del turismo como principal sector de la economía insular a lo que se une la capacidad de arrastre sobre la actividad en otras ramas productivas.

Tras un análisis realizado a empresas catalogadas como de alojamiento turístico y de corta estancia, de acuerdo con la Clasificación Nacional de Actividades Económicas (CNAE-2009), siguiendo un criterio de búsqueda basado en una cifra de negocios mínima de más de 2 millones de euros y más de 10 trabajadores, ambos referidos a 2013, se observó que del total de 199 compañías analizadas, 160 tenían una página de internet y, de éstas, solo 44 publicaban algún tipo de información en la que se manifestaba estar aplicando políticas socialmente responsables. Estas cifras entran dentro de lo previsible, si se tiene en cuenta trabajos como los de Larrinaga et al. (2006), Holcomb et al. (2007), De Grosbois (2012), o Hsieh (2012).

Asimismo, cabe destacar que la información publicada no se recoge generalmente en informes de sostenibilidad o de RSC, sino de manera dispersa y no estandarizada en diferentes secciones de las páginas de internet de las compañías analizadas. Un primer visionado de las mismas parece evidenciar que la información social y ambiental se utiliza esencialmente para mejorar la imagen y no tanto para rendir cuentas de la gestión realizada, dada la escasez de datos cuantitativos aportados. Queda pendiente para un estudio posterior la realización de un análisis de contenido en profundidad de la información publicada, así como la identificación de las razones explicativas de tan bajo índice de divulgación.

Entre las principales conclusiones cabe destacar la importancia del factor tamaño, que guarda una estrecha relación estadísticamente significativa con la divulgación de información sobre RSC: las empresas con mayores valores en las cifras contables consideradas, tienen una mayor propensión a difundir datos acerca de esta materia. Esta vinculación con el tamaño también se observa al considerar variables societarias como el tipo de sociedad mercantil (las sociedades anónimas parecen ser más proclives a publicar frente a las sociedades de responsabilidad limitada) o la pertenencia a grupos empresariales. También unas cifras superiores en indicadores de rendimiento como el margen neto de explotación, la rentabilidad económica (ROA) o la rentabilidad financiera antes de impuestos (ROE) arrojan resultados significativos. La categoría de los establecimientos propiedad de la sociedad (medida a través del sistema de estrellas) o aspectos como la edad del negocio o el endeudamiento no parecen influir en la decisión de publicar.

Tras presentar en esta sección los objetivos del trabajo y las principales conclusiones, en el siguiente apartado se realiza un breve análisis acerca de la publicación de infor- 
mación sobre responsabilidad social corporativa en el sector del alojamiento turístico. Tras describir la metodología utilizada en el apartado tercero, en la sección cuarta se presentan los resultados alcanzados. El trabajo finaliza con las principales conclusiones alcanzadas y la bibliografía citada.

\section{LA COMUNICACIÓN SOBRE RESPONSABILIDAD SOCIAL CORPORATIVA EN EL SECTOR DEL ALOJAMIENTO TURÍSTICO}

Existen muchas definiciones del concepto de responsabilidad social corporativa (RSC). Una de ellas es la ofrecida por la Comisión Europea (2001: 7) como "la integración voluntaria, por parte de las empresas, de las preocupaciones sociales y medioambientales en sus operaciones comerciales y sus relaciones con todos sus interlocutores". Sin entrar a analizar la validez o no de dicha definición, en diversos ámbitos se sostiene que una buena gestión, por ejemplo en el campo ambiental, ayuda a crear una ventaja reputacional con impacto favorable en la gestión financiera de la empresa (Miles y Covin, 2000; Konar y Cohen, 2001; Orlitzky et al., 2003). Incluso, existe un cierto consenso en que la adopción de estrategias de relaciones públicas como la realización de declaraciones públicas, firmas de acuerdos de colaboración y la adopción de compromisos voluntarios pueden tener impactos positivos sobre la imagen de la empresa, provocando un aumento del valor de mercado de las acciones y de la rentabilidad de la empresa (Baker y Sinkula, 2005).

Existe una estrecha conexión entre turismo y RSC, lo cual afecta a un país como España, considerado uno de los principales líderes del sector (Consejo Económico y Social de Canarias, 2013). Nuevos elementos como el desarrollo de modelos alternativos, la aparición de competidores en otros países, la creciente importancia de los problemas ambientales, internet, o los cambios en el perfil del turista, hacen que, como señala Kasim (2006), tras el modelo de crecimiento económico generado por el turismo de masas surja la necesidad de que el negocio turístico sea además socialmente responsable.

Esta preocupación no es una novedad, pues ya desde finales de la década de los setenta el sector había comenzado a abordar esta cuestión y reconocer la importancia de que la actividad turística fuese socialmente responsable como una nueva orientación estratégica. En este sentido se observa un número mayor de empresas hoteleras que incorporan políticas de RSC en su toma de decisiones (Bohdanowicz y Zientara, 2009). Así, y en atención a lo expuesto, la Organización Mundial del Turismo (UNEP y UNWTO, 2005) considera que el desarrollo de un turismo socialmente responsable ha de responder a las necesidades de los turistas y de las regiones anfitrionas presentes, protegiendo y mejorando las oportunidades del futuro. Además, señala que el sector ha de estar enfocado hacia la gestión de todos los recursos de manera que se satisfagan todas las necesidades económicas, sociales y estéticas, y a la vez se respeten la integridad cultural, los procesos ecológicos esenciales, la diversidad biológica y los sistemas de soportes de la vida. En el cuadro 1 se recogen los principios que definen el turismo sostenible. 


\section{Cuadro 1}

\section{LOS PRINCIPIOS DEL TURISMO SOSTENIBLE}

El turismo sostenible debe:

1) Dar un uso óptimo a los recursos medioambientales, que son un elemento fundamental del desarrollo turístico, manteniendo los procesos ecológicos esenciales y ayudando a conservar los recursos naturales y la diversidad biológica.

2) Respetar la autenticidad sociocultural de las comunidades anfitrionas, conservar sus activos culturales y arquitectónicos y sus valores tradicionales, y contribuir al entendimiento y la tolerancia intercultural.

3) Asegurar unas actividades económicas viables a largo plazo, que reporten a todos los agentes, unos beneficios socio-económicos bien distribuidos, entre los que se cuenten oportunidades de empleo estable y de obtención de ingresos y servicios sociales para las comunidades anfitrionas, y que contribuyan a la reducción de la pobreza.

Fuente: (UNEP y UNWTO, 2005, p. 11).

A lo largo del tiempo, tanto instituciones públicas como organismos privados han propuesto diferentes herramientas, protocolos y metodologías con el propósito de que las empresas adopten un compromiso social y ambientalmente responsable. Dichas iniciativas abordan facetas como el medio ambiente, los derechos humanos, las relaciones laborales, etc. Algunas son certificables y otras no pero, en términos generales, se han caracterizado por su voluntariedad. Es el caso de los estándares adoptados por las empresas hoteleras que operan en las Islas Canarias, algunos de los cuales se enuncian a continuación, partiendo de las consultas realizadas a sus páginas de internet:

- Pacto Mundial de las Naciones Unidas (Global Compact)(https://www.unglobalcompact. org)

- Emas (Eco-Management and Audit Scheme, o Reglamento Comunitario de Ecogestión y Ecoauditoría) (http://ec.europa.eu/environment/emas/index_en.htm)

- Serie ISO (International Organization for Standardization) 14000 (http://www.iso. org/iso/isol4000)

- Global Reporting Initiative (GRI) (https://www.globalreporting.org)

- Código ético mundial de la Organización Mundial del Turismo (OMT) (http:// ethics.unwto.org/content/global-code-ethics-tourism)

- Código ECPAT (http://www.ecpat.net)

- Carbon Disclosure Project (CDP) (https://www.cdp.net)

- Travelife (http://www.travelife.info/)

Si bien desde los años setenta del siglo XX hay evidencias de información corporativa de tipo social, es en las décadas de los ochenta y noventa cuando un tipo concreto, el informe ambiental, comienza a ser de uso común entre las grandes empresas en conexión con la implantación de sistemas de gestión ambiental. También a finales del milenio se 
observa un gran interés en que los asuntos sociales pasen a formar parte de la agenda empresarial, debido al aumento de las críticas por mala conducta en temas sensibles como el racismo, la explotación infantil o la corrupción, entre otros. Según un informe elaborado por Ernst and Young (2014), donde se recoge la opinión de 163 inversores institucionales e identifica las tendencias que influyen en las decisiones de inversión, nueve de cada diez inversores valoran la información no financiera (relativa a medio ambiente, sociedad y gobierno corporativo) de las compañías.

Se han publicado diferentes estudios en torno a la relación entre la información de sostenibilidad y el sector hotelero. Así, para Assaf et al. (2012) existe una tendencia por parte de la sociedad a que los hoteles aumenten su compromiso en la publicación de informes siguiendo generalmente el enfoque "Triple bottom line" (TBL) ${ }^{1}$. En el caso que ellos estudian, centrado en Eslovenia, se muestra cómo un mayor volumen de información sobre la materia conduce a un mejor funcionamiento del hotel.

No obstante, en el estudio de Holcomb et al. (2007), quienes analizaron el contenido de los informes anuales y de RSC de los diez primeros hoteles del mundo, destaca que el $80 \%$ de las empresas analizadas centran sus actividades socialmente responsables en donaciones caritativas. Asimismo, el $60 \%$ informó sobre políticas de diversidad y sólo el $40 \%$ proporcionó alguna mención sobre responsabilidad social en su visión o declaraciones de misión. A parecidas conclusiones llega De Grosbois (2012), en su estudio sobre las prácticas de información sobre RSC en las 150 primeras empresas hoteleras del mundo, identificando los métodos de comunicación utilizados y su alcance. Entre otros aspectos, resalta que si bien estas empresas manifiestan un alto grado de compromiso en la materia, pocas indican cómo alcanzar los objetivos propuestos y qué resultados han obtenido. También se señala que a pesar de moverse en el mismo sector, existen diferentes metodologías de análisis y falta de homogeneidad.

También Larrinaga et al. (2006), tras estudiar el sector turístico balear, llegan a la conclusión de que la información ambiental se utiliza para mejorar la imagen de unas organizaciones que no están cambiando profundamente. Aunque se llevan a cabo acciones en el campo ambiental, estas son limitadas. Un aspecto importante es que consideran que la falta de presión del público hace que las empresas analizadas no utilicen la información ambiental para controlar el discurso sobre los temas ambientales.

En el caso concreto de la información publicada en las páginas de internet, Hsieh (2012) estudió las páginas corporativas de los 50 hoteles más importantes del mundo, mediante un análisis de contenido. Se observa que sólo el $46 \%$ de las empresas seleccionadas usaron páginas de internet para mostrar información relacionada con el medio ambiente, destacando los casos de Wyndham, IHG, Accor, Whitbread, Hyatt, Rezidor, Sol Melia, TUI, y Scandic. Dicho autor señala como limitaciones a su estudio que en el momento de realizar la investigación era probable que las empresas hubiesen establecido políticas o iniciados programas ambientales no incluidos en la red. Otro problema es que la información de las páginas de internet puede ser modificada en cualquier momento.

1 Este término, de aplicación a la gestión empresarial, es definido por Elkington (1999, p. 3) como "aquel que se compromete a la búsqueda simultánea de la prosperidad económica, la calidad medioambiental y la justicia social". Bajo esta idea subyace la concepción de que la empresa no se puede centrar exclusivamente en los datos financieros sino que ha de considerar su gestión en equilibrio con los asuntos sociales y ambientales. 


\section{OBJETIVOS Y METODOLOGÍA}

En este apartado se presentan los aspectos metodológicos relacionados con el objetivo propuesto de identificar las variables que influyen en la comunicación del desempeño socialmente responsable en el sector turístico. Para ello se utilizará la información difundida a terceros a través de las páginas de internet corporativas, buscando patrones que permitan establecer relaciones entre dicha información y determinadas características económico-financieras de las empresas consideradas; todo ello en el caso concreto de las empresas de alojamiento hotelero que operan en las Islas Canarias.

Dado que el archipiélago canario es actualmente uno de los principales destinos turísticos no solo a nivel nacional sino también internacional y teniendo en cuenta la estrecha relación entre la actividad turística y la gestión social y ambientalmente responsable, en primer lugar se realizó un análisis de la información publicada en las páginas de internet de las empresas de alojamiento turístico cuyas operaciones se realizan en el archipiélago. El propósito fue identificar las prácticas de divulgación de su desempeño en la materia. El hecho de recurrir a las páginas de internet obedeció a que permiten un acceso más amplio e inmediato a la información que a través de los medios tradicionales, siendo factible contrastar los datos aportados por las empresas. Posteriormente se realizó un análisis estadístico con el objeto de establecer la existencia de relación entre la publicación (o no) de dicha información y la situación económico-financiera de la empresa, medida a través de determinadas variables societarias y contables.

\section{Hipótesis}

Como los contrastes a realizar fueron numerosos, a continuación se presenta de forma genérica la hipótesis de trabajo planteada en el estudio:

$\boldsymbol{H}_{0}$ : No hay diferencias entre las empresas que publican y no publican información sobre RSC para la variable analizada.

\section{Fuentes de los datos}

Para la primera fase del estudio y de entre las diferentes opciones posibles, se consideró por su sencillez y rapidez construir la base de datos de partida a través de una consulta a la aplicación SABI <http://www.einforma.com/sabi> de la empresa Informa SA y gestionada por Bureau van Dijk Electronic Publishing (BvDEP). Además de recoger las cuentas anuales de más de un millón de sociedades españolas, SABI ofrece determinada información de interés para la realización del estudio, como el nombre de la empresa, domicilio, teléfono, fax, en algunos casos su página de internet y, por supuesto, el código de actividad según la Clasificación Nacional de Actividades Económicas en su edición de 2009 (CNAE-2009), esencial para ubicar la empresa dentro de la población objeto de estudio. Se puede considerar que la base de datos SABI es representativa del total de empresas españolas con forma jurídica societaria (excluyendo organismos autónomos) registradas en el Directorio Central de Empresas (DIRCE) (Instituto Nacional de Estadística, 2014), 
cifradas a 1 de enero de 2014 en 1.535 .748 empresas, pues el error medio ${ }^{2}$, partiendo de que el total de empresas españolas activas incluidas en SABI en marzo de 2015 es de 1.366.786, se sitúa en torno al 0,028372\%. Una limitación obvia para el presente estudio es que SABI excluye las cuentas de las empresas bajo la forma de personas físicas, al no ser de depósito obligatorio en el Registro Mercantil.

El criterio de búsqueda consistió en localizar sociedades en activo domiciliadas en Canarias que tuviesen uno o ambos de los códigos CNAE-2009 "551-Hoteles y alojamientos similares" y "552-Alojamientos turísticos y otros alojamientos de corta estancia". Además debían tener un número de empleados mínimo de 10 y una cifra de negocios mínima de 2 millones de euros, ambos durante $2013^{3}$. Esta última restricción tenía como propósito excluir las denominadas micro-empresas ${ }^{4}$. El resultado obtenido fue de 194 empresas.

A continuación se localizaron y consultaron las páginas de internet de dichas empresas al objeto de localizar como mínimo los ítems que se citan a continuación:

- Memorias de sostenibilidad y/o informe de responsabilidad social corporativa.

- Certificaciones de gestión ambiental ISO 14000, EMAS, o similar.

- Certificaciones de seguridad e higiene laboral, OSHAS 18001, o similar.

- Adhesión a códigos de conducta como el pacto mundial de Naciones Unidas (Global Compact) o similares.

- Adhesión a códigos de conducta o similares específicos del sector turístico (ECPAT, WTO, etc.).

Aunque la base de datos SABI incluye las páginas de internet de las empresas registradas, al tratarse de un dato de declaración voluntaria, no todas las compañías lo han hecho, siendo su número escaso. Por tal motivo, fue necesario realizar una búsqueda a través de la aplicación Google, utilizando como clave el nombre de la sociedad y localizar así su página de internet. Los resultados obtenidos se muestran en la siguiente sección.

2 Para calcular el error medio se partió de la expresión

$$
\mathrm{e}=\mathrm{k} \sqrt{\frac{\mathrm{N}-\mathrm{n}}{\mathrm{N}-1} \cdot \frac{\mathrm{p} \cdot \mathrm{q}}{\mathrm{n}}}
$$

siendo e el error de la muestra; $\mathrm{N}$ el tamaño de la población según el DIRCE; $\mathrm{n}$ el tamaño de la muestra (empresas incluidas en la base de datos $\mathrm{SABI}) ; \mathrm{p}=\mathrm{q}=0,5$ ya que, en principio, se desconoce el valor que pueden alcanzar estas variables; $\mathrm{k}=2$, aproximadamente equivalente al 95,5\% del intervalo de confianza de una distribución normal.

3 Debe tenerse en cuenta que la base se actualiza completamente con una periodicidad anual. La búsqueda de empresas con datos referidos a 2014 en la fecha en que se realizó el estudio arrojaría un volumen insignificante. Ello se debe a que, de acuerdo con la legislación española, las empresas tienen como plazo máximo para el depósito de las cuentas anuales de los ejercicios cerrados en diciembre el mes de julio del año posterior.

4 Existen diferentes criterios para clasificar las empresas en función del tamaño; en este caso, se ha optado por seguir la Recomendación 2003/361/CE de la Comisión, de 6 de mayo de 2003 (DOUE L 124/36 de 20.5.2003) sobre la definición de microempresas, pequeñas y medianas empresas, (Comisión Europea, 2003) según la cual se consideran microempresas aquellas empresas que ocupan a menos de 10 personas y cuyo volumen de negocios o cuyo balance general anual no supera los dos millones de euros. 


\section{Variables}

Las variables consideradas en el estudio fueron: la publicación de información sobre RSC (como dependiente), la forma jurídica, la edad, la calidad de los establecimientos, el tamaño, el grupo empresarial, el endeudamiento y la rentabilidad.

\section{Publicación de información sobre RSC}

Es la variable dependiente considerada en el trabajo. Se optó por una variable dicotómica que midiese la publicación o no de datos sobre las prácticas de RSC de la empresa en su página web. Así, se le asignó un valor de «1» si las empresas informaban sobre al menos uno de los ítems propuestos $\mathrm{y} \ll 0 »$ si no incluían ninguna mención al respecto. Si bien se estudió establecer una escala discreta basada en el número de evidencias obtenidas para cada empresa, dicha opción fue descartada ante la dificultad de asignar un peso relativo a cada uno de los ítems informativos en función de su posible impacto económico, social, político o ambiental. Asimismo, procede recordar que el objeto de análisis es la mención en páginas de internet corporativas acerca del desempeño en dicha materia.

\section{Forma jurídica}

Es usual que en los estudios sobre RSC los sujetos de análisis sean sociedades anónimas cotizadas, lo que implica que la forma jurídica no sea un factor relevante a considerar en los mismos; no obstante, las empresas analizadas en este estudio pueden operar bajo la forma de sociedad anónima o la de sociedad limitada. Tras analizar la Ley de Sociedades de Capital vigente en España (regulada por el Real Decreto Legislativo 1/2010), es factible suponer que factores como la posible dispersión de la participación accionarial y la facilidad para desinvertir en el negocio, propios de las sociedades anónimas frente a las sociedades limitadas, hagan que ese tipo de formas jurídicas sean más proclives a adoptar conductas socialmente responsables, pues cuanto más dispersa esté la propiedad, mayores serán las demandas de información por parte de los propietarios. Otro factor a considerar se encuentra en los requisitos de capital: mientras en las sociedades limitadas su cuantía mínima es de 3.000 euros, en el caso de las sociedades anónimas se eleva a 60.000 euros. En el presente trabajo se ha considerado una variable dicotómica descriptiva el tipo de sociedad (anónima o limitada) como factor explicativo de la divulgación o no de información sobre RSC.

\section{Edad}

Es otro posible elemento a considerar pues, según Cochran y Wood (1984, 55-56), aquellas empresas que poseen activos muy antiguos tenderían a ser social y ambientalmente menos sensibles. Una posible causa de tal comportamiento podría hallarse en los elevados costes asociados a la modernización de las instalaciones a fin de cumplir los estándares. Ahora bien, aunque la empresa carezca de problemas con la renovación de equipos, puede suceder que los directivos adopten comportamientos más inflexibles 
a medida que la empresa envejece, al ser más costoso promover cambios drásticos en la estrategia corporativa (Roberts, 1992: 605). En el presente trabajo se ha analizado el inconveniente de que hechos como combinaciones de negocios o cambios de actividad puedan afectar a la determinación de la edad efectiva de la empresa, optándose finalmente por calcular la diferencia en años entre 2013 y la fecha de constitución incluida en la base de datos utilizada en ausencia de otra información alternativa.

\section{Categoría de los establecimientos}

Una primera aproximación a los indicios de calidad hotelera se encuentra en el cumplimiento de determinados criterios (confortabilidad, tamaño de las habitaciones, servicios ofrecidos, etc.) cuyo propósito es establecer la categoría del establecimiento, existiendo sistemas como los basados en letras (A, B, C, D, E), en clases (cuarta, tercera, segunda, primera) o en estrellas (una, dos, tres, cuatro, cinco), entre otros. En España, el sistema utilizado es el de las estrellas, si bien debe tenerse presente que los criterios de asignación pueden variar de un país a otro y, dentro del territorio español, de una comunidad autónoma a otra. Es el caso de las Islas Canarias, cuyos estándares hoteleros se regulan por el Decreto (autonómico) 142/2010, de 4 de octubre. Dada la facilidad para acceder a esta información se optó por utilizar el sistema de categorías. En el caso de que una empresa dispusiera de varios establecimientos alojativos se adoptó la regla de asignarle la mayor categoría alcanzada por cualquiera de ellos. Se optó por este criterio, frente a otros como el valor medio o la mediana, al presumirse que cuanto más alto fuese el indicio de calidad alcanzado por un establecimiento propiedad de la sociedad, mayor debería ser la propensión a informar sobre el desempeño en materia de RSC, considerada en este caso como un elemento más del sistema de gestión de la calidad.

\section{Tamaño}

Puede ser un factor a tener en cuenta al delimitar la susceptibilidad de la empresa para adoptar comportamientos socialmente aceptables e informar sobre ello, debido a que a mayor tamaño, mayor visibilidad y presión social por parte de los grupos de interés relevantes (Patten, 1991; Roberts, 1992: 605; Udayasankar, 2008). También es factible que la empresa pueda tener más accionistas preocupados por las prácticas de RSC así como disponer de un mayor uso de canales formales para difundir su desempeño en la materia (Cowen, et al., 1987). No obstante, el desarrollo de internet puede poner en igualdad de condiciones a grandes y pequeñas empresas a la hora de difundir su desempeño en la materia, pero a la vez, ampliar el escrutinio social a todas las empresas, con independencia de su tamaño.

Spicer (1978), Tilt (1997) o Ahmed et al. (1998), entre otros, establecen que la conciencia ambiental se incrementa con el tamaño de la empresa (medido, entre otras variables, por el número de trabajadores, la cifra de ventas y el total de activos). En este sentido, algunos autores apuntan que, en términos generales, la mayoría de las pequeñas y medianas empresas tienen mayores dificultades en el ámbito de los recursos financieros (SustainAbility, 1997; Henriques y Sadorsky; 1996; Johnson y Greening, 1999) y la 
disposición de un sistema de gestión avanzado (Brammer y Millington, 2006) para acometer proyectos bajo criterios de RSC, llegando a considerarse arriesgados, como señalan Ahmed et al. (1998). Por su parte, García-Sánchez (2008) propone una combinación de falta de recursos y una menor exposición al escrutinio público por parte de estas empresas como factores adversos a la puesta en marcha de acciones en materia de medio ambiente. No obstante, Udayasankar (2008), sugiere un modelo en forma de U, donde las empresas más pequeñas y las más grandes serían más proclives a una política en materia de RSC, con respecto a las empresas medianas, obedeciendo en el primer caso al deseo de ganar visibilidad frente a competidores de mayor tamaño. Para el caso español y según la Fundación Entorno (2001, 2003), las empresas de más de 20 trabajadores son las que tienen una mayor probabilidad de incluir el medio ambiente dentro de su estrategia empresarial; por tal motivo algunos estudios sobre la materia como los de Bravo et al. (2005) o Buil et al. (2005) establecen esa cifra como mínima para seleccionar las empresas que formarán parte de la muestra a analizar.

En el presente trabajo se han utilizado las siguientes magnitudes descriptivas del tamaño: la cifra de negocios, el total de activos, los gastos de personal y el número de trabajadores.

\section{Grupo empresarial}

Esta variable está conectada con el factor tamaño pero también con la existencia de instrumentos de control corporativo. La hipótesis subyacente es que si una empresa forma parte a su vez de un grupo empresarial es factible que la visibilidad del conjunto afecte a cada una de las partes que lo integran como si de una unidad se tratase. Dicha mayor visibilidad puede conducir a que se establezcan instrumentos de control sobre las empresas del grupo. En el presente trabajo se han considerado cuatro medidas relacionadas con la variable grupo empresarial: pertenencia o no a un grupo, número de empresas que forman parte del grupo, tenencia o no de empresas dependientes y número de empresas dependientes. Se han incluido tanto sociedades pertenecientes al negocio del alojamiento de corta estancia como del resto de sectores de actividad, haciéndose además abstracción de los lugares donde opera el resto de empresas del grupo, pues la presunción básica en este caso es suponer que a mayor volumen del conjunto, con independencia de a qué se dediquen sus componentes, más mecanismos de supervisión se implantarán para proteger la imagen corporativa como sujeto socialmente responsable.

\section{Endeudamiento}

Las entidades financieras pueden percibir como un riesgo prestar a empresas con un bajo compromiso en materia de RSC, por lo que reclamarían mayores garantías de cumplimiento a sus deudores (Bryce, 1992). Ello puede deberse a que si los prestatarios soportan no solo multas y sanciones, sino también la falta de confianza de los clientes y consumidores, pueden tener dificultades para hacer frente a la devolución de los préstamos. Pero a la vez, la existencia de una fuerte dependencia de los prestamistas puede limitar la capacidad de la empresa para destinar fondos a actividades socialmente responsables. 
En este sentido, la estructura de capital es un factor donde los acreedores desempeñan un papel relevante (Cornell y Shapiro, 1987; Barton et al., 1989) siendo susceptibles de influir en la capacidad de la empresa para acometer acciones en materia de RSC y rendir cuentas por ello. De ello se deduce que a mayor grado de dependencia de los acreedores para acometer proyectos, mayores serán sus expectativas en torno al papel desempeñado por la empresa en materia de RSC. En este sentido, Roberts (1992) encontró una correlación significativa entre aquellas empresas que informaban sobre su RSC y la ratio de endeudamiento (indicativo del grado de dependencia respecto de los acreedores), entre otras variables. También Andrikopoulos y Kriklani (2013) señalan que la comunicación de información ambiental está relacionada con la estructura financiera de la empresa y que el uso de financiación externa está asociada con problemas de agencia, donde los suministradores de fondos necesitan establecer mecanismos de control para determinar si el agente está usando los recursos de la mejor forma posible. En este sentido y como señala Ullmann (1985), cuanto más relevante sea un grupo de interés más probable es que sus demandas sean satisfechas. A este respecto, se ha observado una relación negativa entre el ratio de endeudamiento y la amplitud de las políticas de RSC y comunicación de la misma (Brammer and Pavelin, 2006).

En el presente trabajo se han considerado como magnitudes representativas del endeudamiento la ratio de endeudamiento y el coste medio de la financiación externa. En el anexo se recogen los métodos de cálculo utilizados para obtener las ratios e indicadores expuestos.

\section{Rendimiento}

Entre los objetivos principales de la empresa se encuentra la generación de beneficios, siendo su evolución en el tiempo un indicador de la gestión realizada por los directivos. Por tal motivo, es factible suponer que, como señala Ullmann (1985: 553), en períodos de bajos rendimientos, en los que la supervivencia de la empresa atraviesa una situación difícil, las demandas de carácter económico tenderán a prevalecer sobre la pretensión de destinar recursos para la mejora de la posición social y ambiental de la empresa, sobre todo cuando los costes asociados son elevados. Según Roberts (1992: 604), la teoría de los stakeholders predice una asociación positiva entre las medidas de desempeño económico y los niveles de revelación de información sobre la RSC. Así, a medida que los beneficios suben es más probable que existan recursos disponibles para desarrollar la actividad bajo criterios de RSC (Adams y Hardwick, 1998; Johnson y Greening, 1999; McGuire et al., 1988; Brammer y Pavelin 2006), al estar menos sujetos a las presiones de otros grupos de interés financieros relevantes como los acreedores (Brammer and Millington, 2006). De esta forma se fortalecen las relaciones de la empresa con sus grupos de interés aunque su impacto en el negocio y por tanto en la riqueza de los accionistas sea a largo plazo (Andrikopoulos y Kriklani, 2013). En estudios como los de Feldman et al. (1997) o Repetto y Dustin (2000) se afirma que las empresas con políticas de RSC pueden tener a la vez resultados financieros aceptables, señalando que incluso puede existir una relación entre neutra o positiva entre el desempeño social y el financiero. Asimismo, en la revisión de 52 trabajos publicados entre los años 70 y 90, realizada por Roman et al. (1999), se observa que 33 muestran la existencia de relación positiva entre ambas variables, 14 señalan una relación neutra, mientras que sólo 5 evidencian una relación negativa. 
No obstante, ello no implica, necesariamente, que por tener una política de RSC la empresa mejore su desempeño financiero, sino probablemente lo expuesto anteriormente: tener beneficios permite disponer de recursos posiblemente ociosos para llevar a cabo dichas políticas. Es el caso del estudio de Simpson y Kohers (2002), realizado con datos del sector bancario: si bien se observa una relación positiva entre la RSC y el desempeño financiero, sus autores no consiguen determinar si las políticas basadas en la RSC favorecen una mejora de los resultados financieros o si es al revés. En el presente trabajo se ha optado por una visión amplia de las medidas descriptivas del rendimiento de la empresa: rentabilidad económica o de los activos, rentabilidad financiera antes de impuestos, margen neto de explotación y margen de beneficios. En el anexo se exponen los métodos de cálculo utilizados para obtener las ratios e indicadores expuestos.

\section{Modelos utilizados}

Una vez clasificadas las empresas en función de los resultados obtenidos, se realizó un análisis estadístico univariante con el propósito de contrastar si la publicación (o no) de información social y ambiental guardaba relación con las medidas descriptivas de las variables consideradas en párrafos anteriores.

En primer lugar, se utilizó la prueba $\chi^{2}$ de independencia para contrastar las variables categóricas. En el caso de variables discretas y continuas, la medida de tendencia central utilizada fue la mediana, debido a que normalmente las muestras elaboradas con datos contables tienden a presentar valores extremos y el uso de la media podría dar lugar a interpretaciones erróneas. Dado que la mediana tiene la ventaja de ser robusta frente a la presencia de valores extremos y que, por otra parte, las muestras no presentaban una distribución normal se utilizó como prueba estadística de contraste el test de WilcoxonMann-Whitney (también denominado U de Mann-Whitney). La herramienta utilizada para el estudio fue el paquete estadístico Stata SE/11 (StataCorp, 2009).

El análisis univariante propuesto no tiene en cuenta las interacciones entre las diferentes variables analizadas y su efecto global sobre la publicación o no de información sobre RSC por parte de las empresas analizadas. Por tal motivo, en una segunda etapa del estudio se trató de identificar aquellas variables que de forma conjunta mejor explicasen la variable dependiente binaria "publicación / no publicación" sobre RSC. Se siguió el modelo de regresión logística o logit (también incluido en la aplicación Stata SE/11) antes que el análisis discriminante al no exigir requisitos de normalidad multivariante e igualdad de matrices de varianzas-covarianzas entre los grupos (Hair et al., 1999, Jovell, 1995). A través de este análisis se puede estimar la matriz de parámetros $\beta$ que conforman la relación de dependencia estadística entre la matriz de variables explicativas X y la probabilidad de que la variable dependiente dicotómica o binaria y adopte el valor 1 , siguiendo la siguiente expresión:

$$
p(y=1)=\frac{e^{\beta_{0}+\beta_{1} x_{1}+\beta_{2} x_{2}+\ldots+\beta_{n} x_{n}}}{1+e^{\beta_{0}+\beta_{1} x_{1}+\beta_{2} x_{2}+\ldots+\beta_{n} x_{n}}}
$$


La principal ventaja de este modelo frente al de regresión lineal es que el resultado del modelo quedará constreñido al intervalo $(0,1)$ y la línea de regresión de la probabilidad predicha tendrá una forma de "s" suavizada, de manera que incrementos elevados de la variable dependiente se corresponderán con incrementos menores de la variable dependiente.

\section{RESULTADOS}

El estudio realizado a continuación sólo considera las empresas que disponen de página de internet. Tras la búsqueda de datos referidos a asuntos sociales y ambientales en las páginas web de las empresas que componían la muestra, de acuerdo con los criterios señalados en la sección anterior, se observó que, del total de 194 compañías hoteleras analizadas, 160 tenía una página de internet y de éstas solo 44 publicaba algún tipo de información sobre la materia. El contenido abarcaba desde declaraciones de política ambiental hasta indicadores de calidad ambiental, pasando por la enumeración de premios y galardones, o la adhesión a códigos de conducta. En la Tabla 1 se presentan los principales estándares declarados por las empresas.

\section{Tabla 1 \\ PRINCIPALES ESTÁNDARES GLOBALES DECLARADOS POR LAS EMPRESAS DEL ESTUDIO}

\begin{tabular}{lr}
\hline \multicolumn{1}{c}{ Estándar } & Número \\
\hline Global Reporting Initiative (GRI) & 8 \\
Código Ético Mundial de la OMT & 13 \\
Principios del Pacto Mundial & 11 \\
Código ECPAT & 13 \\
Travelife & 12 \\
ISO 14001 & 14 \\
EMAS & 9 \\
Carbon Disclosure Project & 4 \\
\hline
\end{tabular}

Pocas aportan datos numéricos que permitan contrastar sus resultados en la materia. Teniendo en cuenta que en algunos trabajos, como los de Holcomb et al. (2007), De Grosbois (2012), Larrinaga et al. (2006) o Hsieh (2012), se señala el bajo reflejo de los asuntos sociales y ambientales en la información publicada por las empresas del sector, el resultado obtenido no se aleja de lo allí indicado. En la Tabla 2 se muestran algunos datos básicos, distinguiendo entre empresas que publican información sobre RSC y las que no, mostrando además las discrepancias existentes entre los valores medios y los medianos. 


\section{Tabla 2 \\ ESTADÍSTICA DESCRIPTIVA BÁSICA}

\begin{tabular}{lrrr}
\hline \multicolumn{1}{c}{ Descriptor } & $\begin{array}{c}\text { Empresas } \\
\text { que SI } \\
\text { publican }\end{array}$ & $\begin{array}{c}\text { Empresas } \\
\text { que NO } \\
\text { publican }\end{array}$ & \multicolumn{1}{c}{$\begin{array}{c}\text { Total } \\
\text { empresas }\end{array}$} \\
\hline Empresas (número) & 44 & 116 & 160 \\
Total de activos (miles de euros) & 1.920 .285 & 3.447 .340 & 5.367 .624 \\
Promedio de activos (miles de euros) & $43.642,83$ & $29.718,44$ & $33.547,65$ \\
Mediana de activos (miles de euros) & $31.651,64$ & $13.486,83$ & $18.615,16$ \\
Cifra de negocios (miles de euros) & $664.834,20$ & 1.022 .516 & 1.687 .351 \\
Promedio cifra de negocios (miles de euros) & $15.109,87$ & $8.814,80$ & $10.545,94$ \\
Mediana cifra de negocios (miles de euros) & $11.853,83$ & $4.696,10$ & $6.269,72$ \\
Trabajadores (número) & 7.529 & 12.230 & 19.759 \\
Promedio de trabajadores (número) & 171,11 & 105,43 & 123,49 \\
Mediana de trabajadores (número) & 134,50 & 70,50 & 85,50 \\
\hline
\end{tabular}

La primera característica distintiva es que, si bien el número de empresas que publican información sobre RSC en sus páginas es menor que el de empresas que no difunden datos al respecto, los valores promedios y medianos de las magnitudes consideradas son mayores. De todo ello se podría deducir la posible existencia de una relación entre el tamaño de la empresa y la difusión de información sobre esta materia, que deberá ser objeto de contraste.

Si se analizan las variables societarias consideradas en la Tabla 3, no parece observarse una relación estadísticamente significativa entre la divulgación de información sobre RSC y la edad de la empresa (al menos para un nivel de significación del 5\%), ni en lo referente a la categoría, medida a través del número de estrellas máximo que poseen los establecimientos propiedad de la compañía. Ahora bien, el hecho de que la empresa forme parte de un grupo o que tenga participadas es estadísticamente significativo. Según la estadística descriptiva, mientras el 32,73\% de las empresas que están incluidas en un grupo publica información sobre RSC frente al 16,00\% de las que son independientes, el 43,10\% de las empresas con filiales a su cargo publican información sobre RSC, frente al 18,63\% de las compañías que no tienen dependientes ${ }^{5}$. En el caso del tipo de sociedad, también parece existir diferencias significativas estadísticamente, pues el 35,29\% de las sociedades anónimas (SA) de la muestra publica información sobre RSC, frente al 21,73\% (que también lo hace) del total de compañías de responsabilidad limitada (SL).

En cuanto a las variables relacionadas con el tamaño, la Tabla 4 muestra una relación estadísticamente significativa entre la publicación de información sobre RSC y magnitudes como el activo, la cifra de negocios, los gastos de personal o el número de trabajadores.

5 Se excluyó del análisis la variable dicotómica alusiva la tenencia o no de participadas por encima de la mediana debido a que, en este caso, el valor de la mediana era igual a cero y los resultados obtenidos eran idénticos a la variable dicotómica filiales_si, que tiene valor 1 si la empresa tiene participadas y cero en caso contrario. 
En este sentido, cabe recordar que las cifras contables de las empresas que publicaron información sobre RSC son mayores que las de las empresas que no han publicado datos sobre esta materia.

\section{Tabla 3}

\section{RELACIÓN ENTRE LA PUBLICACIÓN DE INFORMACIÓN SOBRE RSC Y DETERMINADAS VARIABLES SOCIETARIAS}

\begin{tabular}{|c|c|c|c|c|c|}
\hline Variable & Descripción & $\mathrm{N}$ & Test & Estadístico & P-value \\
\hline edad & Edad (años) & 160 & Wilcoxon test & $-1,706$ & 0,088 \\
\hline edad_p50 & Edad por encima de la mediana (20 años) (si/no) & 160 & Chi test & 2,5975 & 0,107 \\
\hline tiposoc1 & Tipo de sociedad (SL/SA) & 160 & Chi test & 3,6033 & $0,058^{* *}$ \\
\hline estrellas_5 & $\begin{array}{l}\text { Empresa con establecimientos de } 5 \text { estrellas } \\
\text { (máximo) }\end{array}$ & 160 & Chi test & 1,0737 & 0,300 \\
\hline estrellas_4 & $\begin{array}{l}\text { Empresa con establecimientos de } 4 \text { estrellas } \\
\text { (máximo) }\end{array}$ & 160 & Chi test & 0,0000 & 1,000 \\
\hline estrellas_3 & $\begin{array}{l}\text { Empresa con establecimientos de } 3 \text { estrellas } \\
\text { (máximo) }\end{array}$ & 160 & Chi test & 0,0016 & 0,968 \\
\hline grupo_si & Pertenencia a un grupo (sí/no) & 160 & Chi test & 4,8242 & $0,028 * *$ \\
\hline grupo & Empresas del grupo (número) & 160 & Wilcoxon test & $-4,357$ & $0,000 * * *$ \\
\hline grupo_p50 & $\begin{array}{l}\text { Empresas del grupo por encima de la mediana } \\
\text { ( } 2 \text { empresas) (si/no) }\end{array}$ & 160 & Chi test & 20,6536 & $0,000 * * *$ \\
\hline filiales_si & Tenencia de participadas (sí/no) & 160 & Chi test & 11,1101 & $0,001 * * *$ \\
\hline filiales & Empresas participadas (número) & 160 & Wilcoxon test & $-3,114$ & $0,002 * * *$ \\
\hline
\end{tabular}

Tabla 4

RELACIÓN ENTRE LA PUBLICACIÓN DE INFORMACIÓN SOBRE RSC Y LA VARIABLE TAMAÑO

\begin{tabular}{|c|c|c|c|c|c|}
\hline Variable & Descripción & $\mathrm{N}$ & Test & Estadístico & P-value \\
\hline activo & Activo (miles de euros) & 160 & Wilcoxon test & $-3,588$ & $0,000 * * *$ \\
\hline activo_p50 & $\begin{array}{l}\text { Activo sobre la mediana }(18.615,16 \text { miles de } \\
\text { euros) (si/no) }\end{array}$ & 160 & Chi test & 10,1567 & $0,001 * * *$ \\
\hline $\mathrm{cn}$ & Cifra de negocios (miles de euros) & 160 & Wilcoxon test & $-4,162$ & $0,000 * * *$ \\
\hline cn_p50 & $\begin{array}{l}\text { Cifra de negocios sobre la mediana }(6.269,72 \\
\text { miles de euros) (si/no) }\end{array}$ & 160 & Chi test & 15,1724 & $0,000 * * *$ \\
\hline gpers & Gastos de personal (miles de euros) & 160 & Wilcoxon test & $-4,196$ & $0,000 * * *$ \\
\hline gpers_p50 & $\begin{array}{l}\text { Gastos de personal sobre la mediana }(2.201,08 \\
\text { miles de euros) (si/no) }\end{array}$ & 160 & Chi test & 21,1912 & $0,000 * * *$ \\
\hline ntrab & Trabajadores (número) & 160 & Wilcoxon test & $-3,871$ & $0,000 * * *$ \\
\hline ntrab_p50 & $\begin{array}{l}\text { Trabajadores sobre la mediana ( } 88,5 \text { personas) } \\
\text { (si/no) }\end{array}$ & 160 & Chi test & 12,5392 & $0,000 * * *$ \\
\hline
\end{tabular}


Por otra parte, y como se señala en la Tabla 5, no parece existir diferencias significativas entre las empresas estudiadas y las variables relacionadas con el endeudamiento.

Tabla 5

RELACIÓN ENTRE LA PUBLICACIÓN DE INFORMACIÓN SOBRE RSC Y DETERMINADAS RATIOS DE ENDEUDAMIENTO

\begin{tabular}{llcccc}
\hline \multicolumn{1}{c}{ Variable } & \multicolumn{1}{c}{ Descripción } & $\mathrm{N}$ & Test & Estadístico & P-value \\
\hline ratioend & Endeudamiento (ratio) & 160 & Wilcoxon test & 0,783 & 0,433 \\
ratioend_p50 & $\begin{array}{l}\text { Endeudamiento sobre la mediana }(0,69018) \\
\text { (si/no) }\end{array}$ & 160 & Chi test & 0,5016 & 0,479 \\
ratiocmfin & $\begin{array}{l}\text { Coste medio de la financiación externa (ratio) } \\
\text { ratiocmfin_p50 }\end{array}$ & $\begin{array}{l}\text { Coste medio de la financiación externa sobre } \\
\text { la mediana }(0,0068)(\text { si/no) }\end{array}$ & Wilcoxon test & $-0,697$ & 0,486 \\
& 160 & Chi test & 0,0000 & 1,000 \\
\hline$* *$ : significativo al nivel del 5\%; ***: significativo al nivel del 1\% & & & & \\
\hline
\end{tabular}

También parecen observarse resultados significativos cuando se evalúa el rendimiento de la empresa en sus diversas expresiones. Así, de acuerdo con lo expuesto en la Tabla 6 , puede proponerse que existe una relación entre rentabilidad, tanto económica o como financiera, y publicación de información sobre RSC, de forma que las empresas más rentables se caracterizan por aportar datos en torno a esta materia, lo cual obviamente no implica que para ser rentable haya que difundir información.

\section{Tabla 6 \\ RELACIÓN ENTRE LA PUBLICACIÓN DE INFORMACIÓN SOBRE RSC Y EL RENDIMIENTO}

\begin{tabular}{|c|c|c|c|c|c|}
\hline Variable & Descripción & $\mathrm{N}$ & Test & Estadístico & P-value \\
\hline roa & Rentabilidad económica (ratio) & 160 & Wilcoxon test & $-3,548$ & $0,000 * * *$ \\
\hline roa_p50 & $\begin{array}{l}\text { Rentabilidad económica por encima de la } \\
\text { mediana }(0,0098) \text { (si/no) }\end{array}$ & 160 & Chi test & 12,539 & $0,000 * * *$ \\
\hline roe & Rentabilidad financiera antes de impuestos (ratio) & 160 & Wilcoxon test & $-3,315$ & $0,001 * * *$ \\
\hline roe_p50 & $\begin{array}{l}\text { Rentabilidad financiera antes de impuestos por } \\
\text { encima de la mediana }(0,01417))(\mathrm{si} / \mathrm{no})\end{array}$ & 160 & Chi test & 12,539 & $0,000 * * *$ \\
\hline mne & Margen neto de explotación (ratio) & 160 & Wilcoxon test & $-3,726$ & $0,000 * * *$ \\
\hline mne_p50 & $\begin{array}{l}\text { Margen neto de explotación sobre la mediana } \\
(0,1127) \text { (si/no) }\end{array}$ & 160 & Chi test & 18,056 & $0,000 * * *$ \\
\hline mbfo & Margen de beneficios (ratio) & 160 & Wilcoxon test & $-4,135$ & $0,000 * * *$ \\
\hline mbfo_p50 & $\begin{array}{l}\text { Margen de beneficios sobre la mediana }(0,0829) \\
\text { (si/no) }\end{array}$ & 160 & Chi test & 15,172 & $0,000 * * *$ \\
\hline
\end{tabular}


El análisis univariante puede mostrar la relación o no a título individual entre cada una de las variables seleccionadas y la publicación o no de información sobre RSC por parte de las empresas hoteleras domiciliadas en las Islas Canarias. No obstante, puede ser interesante estimar la probabilidad de que dicha difusión tenga lugar considerando el estudio conjunto de varias variables a través de la estimación de un modelo de predicción logit.

A tal efecto se partió de la inspección previa de los coeficientes de correlación de un conjunto de variables identificadas como significativas estadísticamente en el análisis univariante, observándose un elevado número de correlaciones existentes entre los potenciales regresores, fundamentalmente en los relacionados con el tamaño. Por tal motivo, se decidió que el proceso de selección de variables paso a paso (stepwise) para la construcción del modelo se realizase de forma manual en vez de utilizar la opción automatizada ofrecida por la propia aplicación Stata SE/11, dados los resultados incongruentes ofrecidos por ésta en función de si se escogía la alternativa hacia adelante (o de incorporación progresiva) o hacia atrás (o de eliminación progresiva).

Tras obtener varios modelos prometedores, los mismos fueron sometidos a las pruebas estadísticas incluidas en la instrucción fitstat recogida en el paquete SPost (Long y Freese, 2014). Entre ellas se citan los logaritmos de verosimilitud del modelo completo y del modelo sólo con la constante, la prueba LR (o razón de la verosimilitud), el Pseudo $\mathrm{R}^{2} \mathrm{o} \mathrm{R}^{2}$ de McFadden, el AIC (Criterio de información de Akaike) o el BIC (Criterio de información Bayesiano). En la Tabla 7 se representa el modelo identificado como MF1, seleccionado tras superar el proceso de comparación de los AIC y BIC de las diferentes alternativas evaluadas.

Tabla 7

MODELO LOGIT MF1

\begin{tabular}{lrr}
\hline & \multicolumn{1}{c}{$(1)$} & \multicolumn{1}{c}{$(2)$} \\
Variables & logit coeff & \multicolumn{1}{c}{ odds ratios } \\
\hline rsc & $0,9227 * *$ & $2,5161 * *$ \\
tiposoc1 & $(0,4418)$ & $(1,1115)$ \\
& $1,2310 * * *$ & $3,4247 * * *$ \\
grupo_p50 & $(0,4673)$ & $(1,6004)$ \\
& $1,5646 * * *$ & $4,7808 * * *$ \\
gpers_p50 & $(0,4888)$ & $(2,3369)$ \\
& $1,9129 * * *$ & $6,7727 * * *$ \\
mne_p50 & $(0,4769)$ & $(3,2300)$ \\
& $-4,2159 * * *$ & $0,0148 * * *$ \\
Constant & $(0,6774)$ & $(0,0100)$ \\
& 160 & 160 \\
Observations & 52,97 & 52,97 \\
LR chi2(4) & $8,65 \mathrm{e}-11$ & $8,65 \mathrm{e}-11$ \\
Prob $>$ chi2 & 0,281 & 0,281 \\
Pseudo R2 & $-67,62$ & $-67,62$ \\
Log likelihood & & \\
\hline Standard errors in parentheses & \\
$* * *$ p $<0,01, * *$ p $<0,05, * p<0,1$ &
\end{tabular}

Cuadernos de Turismo, 40, (2017), 225-249 
Como se constata en la Tabla 7, las variables que mejor predicen la publicación o no de información sobre RSC por parte de las empresas hoteleras canarias son: tiposoc1, que identifica el tipo de sociedad mercantil (anónima $=1$ o limitada $=0$ ); grupo_p50, que recoge si la empresa pertenece a un grupo cuyo tamaño rebasa la mediana, cifrada en 2 empresas, $($ valor $=1)$ o no $($ valor $=0)$; gpers_p50, que indica si los gastos de personal se encuentran por encima de la mediana, cifrados en 2.201,08 miles de euros (valor $=1$ ) o no (valor $=0)$; y mne_p50, que señala si el margen neto de explotación es superior a la mediana, cuantificado en el 11,27\% (valor $=1$ ) o no (valor $=0$ ). En resumidas cuentas, factores como la forma jurídica, la adscripción a un grupo empresarial (indicativo de la posible existencia de instrumentos directos de dirección y control sobre la propia compañía necesarios para salvaguardar la reputación del grupo en su conjunto), el tamaño (medido a través de los gastos de personal) y la capacidad de generar rendimientos podrían estar afectando a la publicación de información sobre RSC.

La estimación de la contribución relativa de cada variable se observa en la columna de odd ratios, siendo todos los valores calculados mayores que uno. Esto implica que un aumento en una unidad en el valor de la variable independiente produce a su vez un incremento en la proporción que se indica en la variable dependiente. Así, el hecho de ser sociedad anónima (tiposoc1 $=1$ ) hace que la razón de publicar sobre RSC aumente 2,52 veces, mientras que tener un margen neto de explotación por encima de la mediana supone que dicha razón aumente 6,77 veces.

Con la herramienta SPost de Stata SE/11 se analizó cómo afectaba la variación de una o varias covariables en la predicción de publicar información sobre RSC. Un análisis individualizado, variable a variable, muestra que el hecho de ser sociedad anónima supone que la probabilidad de publicar sobre RSC en la website corporativa aumente en 14,46 puntos porcentuales, respecto de ser sociedad limitada, pues en este caso la probabilidad de publicar sólo es del 13,18\%. Por su parte, la pertenencia a un grupo empresarial formado por más de 2 empresas también influye favorablemente: si la empresa se encuentra igual o por debajo de esta cifra su probabilidad de publicar es del $11,06 \%$, en caso contrario dicha probabilidad llega hasta el $29,86 \%$ (un incremento de 18,80 puntos). Para los gastos de personal, si estos se encuentran por encima de la mediana, establecida en 2.201 miles de euros, la probabilidad de publicar es del 32,95\%, mientras que si es igual o inferior, se reduce al 9,32\% (una diferencia de 23,63 puntos porcentuales). Finalmente y en lo que respecta al margen neto económico, la probabilidad de publicar encontrándose igual o por debajo de la mediana (cifrada en el 11,27\%) es del 7,95\%, mientras que en el caso contrario la probabilidad es del 36,91\% (28,96 puntos de diferencia).

Si por el contrario, se considerasen todas las variables de forma conjunta, en el caso hipotético de que la empresa fuese sociedad limitada; fuese independiente o en todo caso el tamaño del grupo no fuese superior a 2; y ni los gastos de personal ni el margen de explotación rebasasen las medianas respectivas de la muestra analizada (es decir que cada uno de los valores de las variables dicotómicas explicativas consideradas fuese igual a cero), la probabilidad de publicar sería del 1,45\% (el valor de la constante). Por el contrario, si se tratase de una sociedad anónima que formase parte de un grupo con más de dos empresas y cuyos gastos de personal y margen de explotación fuesen 
superiores a la mediana (dicho de otra forma, que cada uno de los valores de las variables dicotómicas explicativas consideradas fuese igual a 1), entonces la probabilidad de publicar sería del $80,46 \%$.

\section{PRINCIPALES CONCLUSIONES}

Si se considera el total de empresas analizadas en el estudio por cumplir el requisito de tener al menos diez trabajadores en plantilla, más de dos millones de euros de cifra de negocios y página de internet (160 compañías), apenas el 27,50\% recogía en su website información accesible sobre asuntos sociales y ambientales. No obstante su escaso peso numérico, dichas empresas poseen unos activos totales por un valor aproximado de 1.920 millones de euros, una cifra de negocios de 645 millones de euros y más de 7.500 trabajadores en plantilla, siendo en términos generales empresas de mayor tamaño que las que no publican información sobre la materia.

La información se presenta de manera dispersa y no estandarizada en diferentes secciones de las páginas de internet de las compañías analizadas. Un primer visionado de las mismas podría evidenciar que la información social y ambiental se utiliza esencialmente para mejorar la imagen y no tanto para rendir cuentas de la gestión realizada, pues apenas se aportan datos numéricos de contraste. Queda pendiente para un estudio posterior la realización de un análisis de contenido en profundidad de la información publicada, así como la identificación de las razones explicativas de tan bajo índice de divulgación.

El factor tamaño parece guardar una estrecha relación con la divulgación de información sobre RSC: a mayores valores de las cifras contables consideradas (total activo y cifra de negocios), mayor propensión a difundir datos acerca de esta materia. Lo mismo sucede con los gastos de personal y el número de trabajadores. Puede establecerse una vinculación con el tamaño si se consideran variables societarias como el tipo de sociedad mercantil (anónima o limitada) o la tenencia de participadas. Formar parte de un grupo parece ser estadísticamente significativo, no así la mayor o menor edad de la empresa.

En lo que respecta al endeudamiento, no parece ser un factor distintivo, probablemente porque se está analizando un sector concreto que se mueve en las mismas coordenadas en esta materia. Sin embargo, los indicadores de rentabilidad económica y financiera así como el margen neto de explotación y el margen del resultado sobre ventas arrojan resultados significativos, existiendo por tanto una relación con la publicación de información sobre RSC.

En cuanto a la probabilidad de publicar información sobre RSC, las variables estadísticamente significativas que estarían influyendo en la decisión son: la forma jurídica (es más probable publicar si se es sociedad anónima), la adscripción a un grupo empresarial (indicativo de la posible existencia de instrumentos directos de dirección y control sobre la propia compañía necesarios para salvaguardar la reputación del grupo en su conjunto), el tamaño (medido a través de los gastos de personal) y la capacidad de generar rendimientos.

Entre las principales limitaciones del presente estudio cabe reseñar el requisito esencial de centrar el análisis en un canal de comunicación específico como es internet, lo que conlleva la exclusión de empresas que, realizando acciones en materia de RSC, no utilicen 
dicho medio y hayan optado por divulgarlas por otras vías, como memorias y folletos impresos o comunicados de prensa o, simplemente, por no informar al respecto. Por otra parte, es posible que en el momento de realizar el estudio la compañía estuviese poniendo en marcha un programa de RSC y, por tanto, no dispusiera de resultados susceptibles de ser presentados. Finalmente, cabe señalar la posibilidad de que las prácticas de RSC comunicadas no se correspondan con el desempeño real en la materia. El hecho de que, para algunos de los ítems analizados, la auditoría no sea legalmente obligatoria y que los autores del presente estudio no hayan tenido acceso a las evidencias custodiadas por las empresas puede afectar a la fiabilidad e interpretación de los datos.

\section{ANEXO}

\section{MÉTODOS DE CÁLCULO DE LAS RATIOS E INDICADORES UTILIZADOS EN EL ESTUDIO}

\begin{tabular}{|c|c|}
\hline Denominación & Expresión \\
\hline \multirow{2}{*}{ Endeudamiento (ratio) } & \multirow{2}{*}{$\frac{\text { Pasivo corriente }_{n}+\text { Pasivo no corriente }_{n}}{\text { Patrimonio neto }_{n}}$} \\
\hline & \\
\hline \multirow{5}{*}{$\begin{array}{l}\text { Coste medio de la financiación } \\
\text { externa (ratio) }\end{array}$} & - Gastos financieros $_{n}$ \\
\hline & (Deudas a largo plazo no comerciales ${ }_{n}+$ ) \\
\hline & Deudas a largo plazo no comerciales ${ }_{n-1}+$ \\
\hline & Deudas a corto plazo no comerciales ${ }_{n}+$ \\
\hline & Deudas a corto plazo no comerciales ${ }_{n+1}$ \\
\hline \multirow{2}{*}{$\begin{array}{l}\text { Rentabilidad económica (ROA) } \\
\text { (ratio) }\end{array}$} & Resultado de explotación $_{n}$ \\
\hline & $\left(\right.$ Activo $_{n}+$ Activo $\left._{n-1}\right) \div 2$ \\
\hline \multirow{2}{*}{$\begin{array}{l}\text { Rentabilidad financiera antes de } \\
\text { impuestos (ROE) (ratio) }\end{array}$} & Resultado antes de impuestos $_{n}$ \\
\hline & $\left(\right.$ Fondos propios $_{n}+$ Fondos propios $\left._{n-1}\right) \div 2$ \\
\hline \multirow{2}{*}{ Margen neto de explotación (ratio) } & Re sultado de explotación $n_{n}$ \\
\hline & Cifra de negocios $s_{n}$ \\
\hline \multirow{2}{*}{ Margen de beneficios (ratio) } & Re sultado del ejercicio ${ }_{n}$ \\
\hline & Cifra de negocios $_{n}$ \\
\hline
\end{tabular}

\section{BIBLIOGRAFÍA}

ADAMS, M.; HARDWICK, P. (1998): “An analysis of corporate donations. United Kingdom evidence", Journal of Management Studies, 35(5), pp. 641-654.

AHMED, N.U.; MONTAGNO, R.V.; FIRENZE, R.J. (1998): "Organizational performance and environmental consciousness: an empirical study", Management Decision, 36(2), pp. 57-62.

ALBAREDA, V. L.; BALAGUER, F.M. R. (2008): “La responsabilidad social de la empresa y los resultados financieros", Revista de Contabilidad y Dirección, 7, pp. 11-26. 
ALIANZA PARA LA EXCELENCIA TURÍSTICA (EXCELTUR) (2014): “Impactur 2013. Estudio del impacto económico del turismo sobre la economía y el empleo en las islas canarias". Recuperado el 15 de diciembre de 2015 de: http://www.gobiernodecanarias.org/opencms8/export/sites/presidencia/turismo/downloads/estadistica/file/2014/ IMPACTUR_Canarias_2013.pdf

ANDRIKOPOULOS, A.; KRIKLANI, N. (2013): "Environmental disclosure and financial characteristics of the firm: the case of Denmark", Corporate Social Responsibility and Environmental Management, 20, pp. 55-64. DOI. 10.1002/csr.1281

ASSAF, A.G.; JOSIASSEN, A.; KNEZEVIC L. (2012): "Does Triple Bottom Line reporting improve hotel performance?", International Journal of Hospitality Management, 31, pp. 596-600.

BAKER, W.E.; SINKULA, J.M. (2005): "Environmental marketing strategy and firm performance. effects on new product performance and market share", Journal of the Academy of Marketing Science, 33(4), 461-475.

BARTON, S.; HILL, N.; SUNDARAM, S. (1989): “An empirical test of stakeholder predictions of capital structure", Financial Management (Spring), pp. 36-44.

BOHDANOWICZ, P.; ZIENTARA, P. (2009): "Hotel companies' contribution to improving the quality of life of local communities and the well-being of their employees", Tourism and Hospitality Research, 9(2), pp. 147-158.

BRAMMER, S.; MILLINGTON, A. (2005): "Corporate reputation and philanthropy: An empirical analysis", Journal of Business Ethics, 61(1), pp. 29-44.

BRAMMER, S.; MILLINGTON, A. (2006): "Firm size, organizational visibility and corporate philanthropy. an empirical analysis", Business Ethics. An European Review, 15(1), pp. 6-18.

BRAMMER, S.; PAVELIN, S. (2006): "Voluntary environmental disclosures by large UK companies", Journal of Business Finance and Accounting, 33(7-8), pp. 1168-1188. doi. 10.1111/j.1468-5957.2006.00598.x

BRAVO GIL, R.; FRAJ ANDRÉS, E.; MARTÍNEZ SALINAS, E. (2005): “La importancia del factor medioambiental en las estrategias corporativas y de marketing. una aplicación al sector de bienes de consumo", Cuadernos de Estudios Empresariales, 15, pp. 199-224.

BRYCE, A. (1992): "Environmental liabilities. Practical issues for lenders", Journal of International Banking Law, 7(4), pp. 131-137.

BUIL CARRASCO, I.; FRAJ ANDRÉS, E.; MARTÍNEZ SALINAS, E.; MATUTE VALLEJO, J. (2005): "Tipología de empresas ambientales en el sector de bienes de consumo final”, Revista de Economía y Empresa, 23 (54/55), pp. 69-93.

COCHRAN, P. L.; WOOD, R. A. (1984): "Corporate social responsibility and financial performance", Academy of Management Journal, 27 (1), pp. 42-56.

COMISIÓN EUROPEA (2001): Libro Verde Fomentar un Marco Europeo para la Responsabilidad Social de las Empresas. Recuperado el 26 de marzo de 2014 de: http:// eur-lex.europa.eu/legal-content/ES/TXT/?uri=CELEX:52001DC0366

COMISIÓN EUROPEA (2003): Recomendación 2003/361/CE de la Comisión, de 6 de mayo de 2003 (DOUE L 124/36 de 20.5.2003) sobre la definición de microempresas, pequeñas y medianas empresas. 
CONSEJO ECONÓMICO Y SOCIAL DE CANARIAS (2013): Informe anual 2013 del Consejo sobre la situación económica, social y laboral de Canarias en el año 2012. Las Palmas de Gran Canaria: CES.

CORNELL, B.; SHAPIRO, A. (1987): "Corporate stakeholders and corporate finance", Financial Management, 16(1). pp. 5-14.

COWEN, S.S.; FERRERI, L.B.; PARKER, L.D. (1987): “The impact of corporate characteristics on social responsibility disclosure. A typology and frequency-based analysis", Accounting, Organizations and Society, 12(2), pp. 111-122.

DE GROSBOIS, D. (2012): “Corporate social responsibility reporting by the global hotel industry: Commitment, initiatives and performance”, International Journal of Hospitality Management, 31, pp. 896-905.

DECRETO 142/2010, de 4 de octubre (BOC de 15 de octubre), por el que se aprueba el Reglamento de la Actividad Turística de Alojamiento y se modifica el Decreto 10/2001, de 22 de enero, por el que se regulan los estándares turísticos.

ELKINGTON, J. (1999): Cannibals with Forks: The Triple Bottom Line of 21st Century Business. Oxford: Capstone.

ERNST AND YOUNG (2014): Tomorrow's investment rules. Global survey of institutional investors on non-financial performance. London: Ernst and Young.

FELDMAN, S.J.; SOYKA, P.A.; AMEER, P.G. (1997): “Does improving a firm's environmental management system and environmental performance result in a higher stock price?" The Journal of Investing, 6(4), pp. 87-97.

FORÉTICA (2011): Informe Forética 2011. Evolución de la responsabilidad social de las empresas en España. Recuperado el 26 de mayo de 2014 de: http://www.foretica.org/ biblioteca/informes-foretica/doc_details/298-informe-foretica-2011-version-extendida?lang=es

FUNDACIÓN ENTORNO (2001): Informe 2001 de la gestión medioambiental en la empresa española. Madrid. Fundación Entorno, Empresa y Medio Ambiente.

FUNDACIÓN ENTORNO (2003): Entorno 2003. Informe sobre la gestión ambiental en la empresa española. Avances hacia la sostenibilidad. Madrid. Fundación Entorno, Empresa y Medio Ambiente.

GARCÍA-SÁNCHEZ, I.M. (2008): "Corporate social reporting. segmentation and characterization of Spanish companies", Corporate Social Responsibility and Environmental Management, 15, pp. 187-198.

GUERRA, A.; FERNÁNDEZ, E. (2011): "Empresas turísticas en España totalmente responsables", CULTUR. Revista de Cultura y Turismo, 5 (2), pp. 43-60.

HAIR, J.F.; ANDERSON, R.E.; TATHAM, R.L.; BLACK, W.C. (1999): Análisis multivariante (5a.ed). Madrid: Prentice Hall Iberia.

HENRIQUES, I.; SADORSKY, P. (1996): “The determinants of an environmentally responsible firm. An empirical approach”, Journal of Environmental Economics and Management, 30, pp. 381-395.

HOLCOMB, J.L., UPCHURCH, R.S.; OKUMUS, F. (2007): “Corporate social responsibility: what are top hotel companies reporting?", International Journal of Contemporary Hospitality Management, 19(6), pp. 461-475. 
HSIEH, Y.C. (2012): “Hotel companies' environmental policies and practices: a content analysis of their web pages", International Journal of Contemporary Hospitality Management, 24(1), pp. 97-121.

INSTITUTO NACIONAL DE ESTADÍSTICA (2014): Estructura y dinamismo del tejido empresarial en España - Directorio Central de Empresas (DIRCE) a 1 de enero de 2014. Madrid: INE. Recuperado el 20 de noviembre de 2014 de: http://www.ine.es/ prensa/np858.pdf

JOHNSON, R.A.; GREENING, D.W. (1999): "The effects of corporate governance and institutional ownership types on corporate social performance", Academy of Management Journal, 42(5), pp. 564-577.

JOVELL, A.J. (1995): Análisis de regresión logística. Cuadernos metodológicos 15. Madrid: Centro de Investigaciones Sociológicas.

KASIM, A. (2006): "The need for business environmental and social responsibility in the tourism industry", International Journal of Hospitality and Tourism Administration, 7(1), pp. 1-22.

KONAR, S.; COHEN, M.A. (2001): "Does the market value environmental performance?" The Review of Economics and Statistics, 83(2), 281-289.

LARRINAGA, C; LLULL, A.; PERELLÓ, M. (2006): "El papel de la información medioambiental en el cambio organizativo: el caso del sector turístico balear", Revista Española de Financiación y Contabilidad, 35(130), pp. 501-522.

LEE, S.; PARK, S.Y. (2009): "Do socially responsible activities help hotels and casinos achieve their financial goals? International Journal of Hospitality Management, 28(1), pp. 105-112.

LONG, J.S.; FREESE, J. (2014): Regression models for categorical dependent variables using Stata, 3rd Edition. College Station, TX: Stata Press.

MCGUIRE, J.B.; SUNDGREN, A.; SCHNEEWEISS, T. (1988): "Corporate social responsibility and firm financial performance", Academy of Management Journal, 31(4), pp. 854-872.

MILES, M.P.; COVIN, J.G. (2000): "Environmental marketing. A source of reputational, competitive, and financial advantage", Journal of Business Ethics, 23, 299-311.

MINISTERIO DE INDUSTRIA, TURISMO Y COMERCIO (2007): Plan del Turismo Español Horizonte 2020, Recuperado el 28 de Mayo de 2014 de. http://www.tourspain.es/eses/VDE/Documentos\%20Vision\%20Destino\%20Espaa/Plan_Turismo_ Espa\%C3\%B1ol_Horizonte_2020.pdf

ORLITZKY, M.; SCHMIDT, F.L.; RYNES, S.L. (2003): “Corporate social and financial performance. A meta-analysis", Organization Studies, 24(3), 403-441.

PATTEN, D.M. (1991): "Exposure, legitimacy, and social disclosure", Journal of Accounting and Public Policy, 10(4), pp. 297-308.

REAL DECRETO LEGISLATIVO 1/2010, de 2 de julio (BOE de 3 de julio), por el que se aprueba el texto refundido de la Ley de Sociedades de Capital. https.//www.boe.es/ buscar/act.php?id=BOE-A-2010-10544

REPETTO, R.; DUSTIN, D. (2000): Pure profit. The financial implications of environmental performance. Washington: World Resources Institute, <http./pdf.wri.org/ pureprofit.pdf> 
ROBERTS, R.W. (1992): «Determinants of corporate social responsibility disclosure: an application of stakeholder theory», Accounting, Organizations and Society, 17(6), pp.595-612. doi.10.1016/0361-3682(92)90015-K

ROMAN, R.M.; HAYIBOR, S.; AGLE, B.R. (1999): "The relationship between social and financial performance. Repainting a portrait”, Business \& Society, 38(1), pp. 109-125. doi.10.1177/000765039903800105

SIMPSON, W.G.; KOHERS, T. (2002): The link between corporate social and financial performance. Evidence from the banking industry", Journal of Business Ethics, 35(2), pp. 97-109.

SPICER, B.H. (1978): "Corporate social performance and information disclosure. An empirical study", The Accounting Review, 53(1), pp. 94-111.

STATACORP. (2009): Stata Statistical Software: Release 11. College Station, TX: StataCorp LP.

SUSTAINABILITY, LTD. (1997): The SME CER. Should small and medium-sized enterprises (SMES) produce company environmental reports (CERS)?. Paper for the Environmental European Agency, June.

TILT, C.A. (1997): "Environmental policies of major companies. Australian evidence", British Accounting Review, 29, pp. 367-394.

UDAYASANKAR, K. (2008): "Corporate social responsibility and firm size", Journal of Business Ethics, 83(2), pp. 167-175.

ULLMANN, A. (1985): "Data in search of a theory: a critical examination of the relationships among social performance, social disclosure, and economic performance of U.S. firms", Academy of Management Review, 10 (3), 540-547.

UNEP and UNWTO (2005): Making Tourism More Sustainable - A Guide for Policy Makers. Paris and Madrid: UNEP and UNWTO.

WORLD TOURISM ORGANIZATION (2016): Compendium of tourism statistics dataset. Madrid: UNWTO. Recuperado el 03 de marzo de 2017 de http://www.e-unwto.org/ toc/unwtotfb/current 
